\title{
Effects of High-Order Laser Distortion Products in Radio over Free-Space Optical Links
}

\author{
G. S. D. Gordon, M. J. Crisp, R. V. Penty and I. H. White \\ Centre for Photonic Systems, Electrical Engineering Division \\ Department of Engineering, University of Cambridge, 9 JJ Thomson Avenue, Cambridge CB3 OFA, UK \\ E-mail: gsdg2@cam.ac.uk
}

\begin{abstract}
RoFSO links are found to be susceptible to high-order laser distortion making conventional SFDR ineffective as a performance indicator. For the first time, peak input power is demonstrated as a service-independent bound on dynamic range.

OCIS codes: (060.2605) Free-space optical communication; (060.5625) Radio frequency photonics.
\end{abstract}

\section{Introduction}

Demand for high-speed wireless data services is constantly increasing meaning that radio coverage and signal quality must keep improving, particularly in traditionally problematic indoor environments. Radio over fiber (RoF) technology has been demonstrated as a cost-effective and high-performance means of distributing broadband, multiservice analog radio signals to remote antennas for improved radio coverage in a distributed antenna system $(D A S)$ [1]. Installing fiber for such systems can be unattractive in certain locations because of cost and practicality and so here it is desirable to replace RoF links with radio over free-space optical (RoFSO) links. This is particularly the case in large internal spaces such as atria or covered sporting venues. Such systems have been proposed for narrowband single-service cellular applications [2], and also for wideband multiservice applications [3].

\section{RoFSO links}

In order to be cost-effective, such RoFSO links must be cheaper than RoF links and so should use minimal extra equipment. These systems should therefore allow for manual line-of-sight alignment and be tolerant to misalignment error. This is often achieved by using divergent beams, which necessarily results in optical power loss due to the limited aperture of the receive optics. There are also unavoidable free-space coupling losses, experimentally shown to be at least $8 \mathrm{dBo}$. Over a distance of $50 \mathrm{~m}$, the minimum useful length of a RoFSO link, it is difficult to reduce optical loss to less than $10 \mathrm{dBo}$, and practical systems often have rather more. To mitigate this high loss and maintain a reasonable power budget, high laser transmit powers are used. In order to keep costs to a minimum, optical amplifiers and external modulators are undesirable. Consequently, a low-cost system is realized by direct intensity modulation of a semiconductor laser at a high CW output power. In order to maximize dynamic range, this laser should be as linear as possible. This provides an extreme set of operating conditions for such RoFSO links, which are not encountered in more conventional RoF systems.

\section{SFDR performance}

To evaluate the performance of such RoFSO links, an experimental link was set up using a laser, a fiber attenuator to simulate free-space loss and a photodiode receiver. The initial laser used for testing was a 1310nm Sumitomo SLV521A DFB operated at a bias current of 50mA with a $10 \mathrm{~mW} \mathrm{CW}$ output. The optical loss used was $11.9 \mathrm{dBo}$. In evaluating the performance of this link, a standard two-tone linearity test was performed to measure the spuriousfree dynamic range (SFDR). For this laser it was found that the first intermodulation distortion (IMD) product, used to gauge linearity, was best approximated using a ninth-order slope. By contrast, in RoF systems third-order effects are usually dominant. This is illustrated in figure 1 and experimental results are presented in figure 2. Similar results showing high-order effects were obtained for two other lasers run at different transmit powers and wavelengths.

(a) Comparison of $3^{\text {rd }}$ order and high order two-tone test IMD power curves

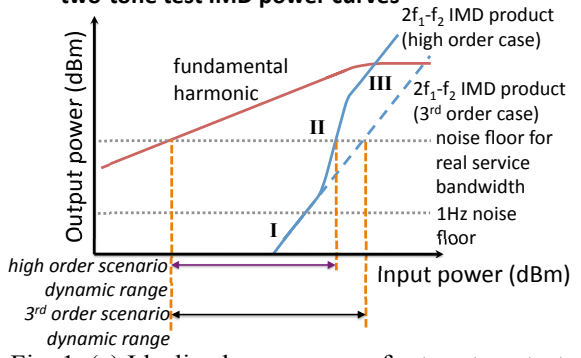

(b)

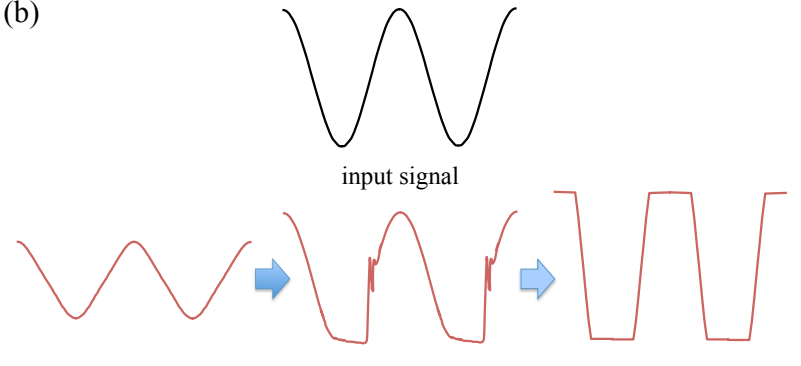

I - static distortion II - dynamic clipping III - static clipping

Fig. 1. (a) Idealized power curve for two-tone test over a RoFSO link with labels indicating different regimes of distortion production as detailed in (b). 
The initial third-order distortion observed in regime I of figure 1 is a result of the intrinsically nonlinear nature of the laser and is referred to as static distortion. This arises from a range of nonlinear effects including gain saturation, spatial hole burning and indeed external effects such as optical feedback. The high-order distortion observed in regime II of figure 1 is believed to be caused by dynamic clipping or dynamic distortion, a result of the laser turn-on delay triggering relaxation oscillations when modulating currents fall close to the threshold [4]. This can be predicted using a dynamic L-I curve, which is generated by pairing input current values with instantaneous output light power values. The dynamic L-I curve for the first laser tested is shown in figure 3. Clearly, this curve would require dominant high-order terms in a Taylor or Volterra series expansion, which explains why high-order effects are so prominent in this regime - a link that has not been made clear previously. Because the lasers are optimized for linearity and the optical losses are high, the onset of dynamic clipping occurs before the static distortion effects are detectable. It begins close enough to the $200 \mathrm{kHz}$ resolution bandwidth instrument noise floor that for services of higher bandwidth it would be the first significant nonlinear effect to occur.

As the modulation power is further increased, the turn-on delay and relaxation oscillations become negligible and the output approaches a clipped sinusoid as shown in regime III of figure 1. The series expansion for this is third-order dominated, explaining why the IMD slope decreases at this point. The different regimes of distortion mean that the slope of the first IMD product power is not constant. As a result, SFDR normalized to a $1 \mathrm{~Hz}$ bandwidth becomes a much less meaningful measure of dynamic range as it cannot be extrapolated to calculate the distortion-free dynamic range of higher bandwidth services. It is desirable to avoid driving the laser so hard as to encounter high-order distortion but it may be necessary to operate close to this point to meet the large dynamic range requirements of wireless services. A reliable means of quantifying this point is needed so that it may be avoided.

\section{EVM performance}

The error vector magnitude (EVM) for three different modulated services $(802.11 \mathrm{~g}, 802.11 \mathrm{~b}$ and $3 \mathrm{GPP}$ QPSK) was measured experimentally over this link and standard comparable measures of peak power were used to compare between them. It was found that there is a clear peak power level around which all the services experience an abrupt increase in EVM due to the onset of this high-order distortion, with the power levels of the EVM minima, or knee points, all occurring within $4 \mathrm{~dB}$ of one another. In a third-order limited system the spread of the knee points was found to be about $7 \mathrm{~dB}$ as shown in figure 2 . The high-order distortion compresses the range of the knee points allowing a single power to better specify the expected peak input power for the onset of distortion, independent of the RF service.
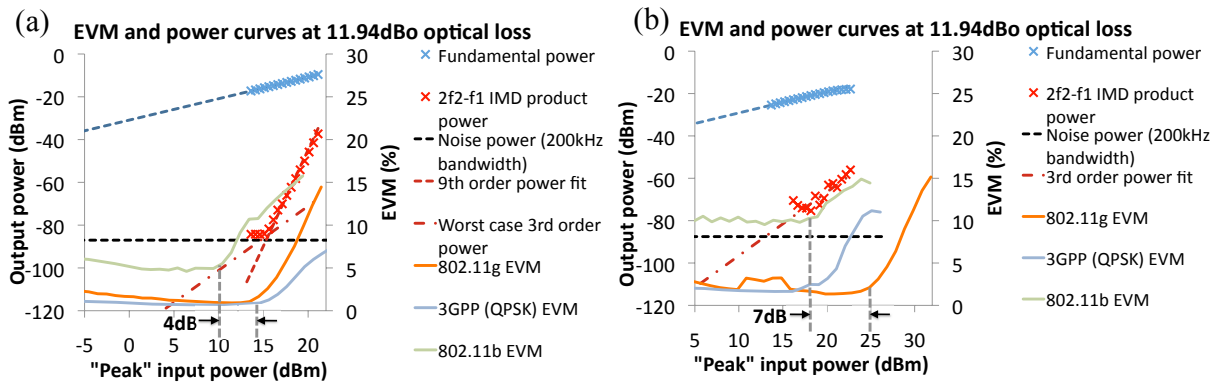

Fig. 2. EVM and power curves for (a) a high-order limited laser, (b) a third-order limited laser.

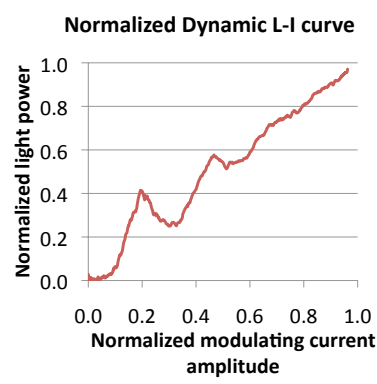

Fig. 3. Dynamic L-I curve.

\section{Conclusions}

The unique high optical loss, high power operating conditions of RoFSO links mean that their dynamic range is often limited by high-order nonlinear effects, a result of dynamic clipping. Consequently, SFDR is no longer a viable service-independent performance measure. Instead, it is found that when EVM for different services is compared a sharp increase is observed within a $4 \mathrm{~dB}$ range of input peak powers, irrespective of the RF service. This represents a $3 \mathrm{~dB}$ compression of this input peak power range compared to a third-order limited case. In RoFSO links this peak power level could be used as a service-independent measure of maximum useful RF input power.

\section{References}

[1] P. Hartmann, X. Qian, R. Penty, and I. White, "Broadband multimode fibre (MMF) based IEEE $802.11 \mathrm{a} / \mathrm{b} / \mathrm{g}$ WLAN distribution system," in 2004 IEEE International Topical Meeting on Microwave Photonics, (IEEE, New York, 2004), pp. 173-176.

[2] H.H. Refai, "Transporting RF signals over free-space optical links," Proceedings of SPIE 5712, 46-54 (2005).

[3] G.S. Gordon, M.J. Crisp, R.V. Penty, and I.H. White, "Demonstration of a Low-Cost Broadband Radio over Free- Space Optics System," in Semiconductor and Integrated Optoelectronics '10, (Cardiff, 2010).

[4] B.H. Wang and W.I. Way, "Large-signal spurious-free dynamic range due to static and dynamic clipping in direct and external modulation systems,” Journal of Lightwave Technology 16, 1773-1785 (1998). 